\title{
Interpretation and resolution of pinch singularities in non-equilibrium quantum field theory
}

\author{
Carsten Greiner* and Stefan Leupold ${ }^{\ddagger}$ \\ Institut für Theoretische Physik, Universität Giessen, \\ D-35392 Giessen, Germany
}

(April 1998)

\begin{abstract}
Ill-defined pinch singularities arising in a perturbative expansion in out of equilibrium quantum field theory have a natural analogue to standard scattering theory. We explicitly demonstrate that the occurrence of such terms is directly related to Fermi's golden rule known from elementary scattering theory and is thus of no mystery. We further argue that within the process of thermalization of a plasma one has to resum such contributions to all orders as the process itself is of non-perturbative nature. In this way the resummed propagators obtain a finite width. Within the Markov approximation of kinetic theory the actual phase space distribution at a given time of the evolution enters explicitly.
\end{abstract}

PACS numbers: 05.20.Dd, 05.30.-d, 11.10.Wx

Keywords: Non-equilibrium quantum field theory; pinch singularities; transport theory

*e-mail address: carsten.greiner@theo.physik.uni-giessen.de

${ }^{\ddagger}$ e-mail address: stefan.k.leupold@theo.physik.uni-giessen.de 
Non-equilibrium many-body theory or quantum field theory has become a major topic of research for describing various transport processes in nuclear physics, in cosmological particle physics or more generally in quantum dissipative systems. A very powerful diagrammatic tool is given by the 'Schwinger-Keldysh' [1 3] or 'closed time path' (CTP) technique by means of non-equilibrium Green's functions for describing a quantum system also beyond thermal equilibrium 畑. For an equilibrium situation this technique is equivalent to the real time description of finite temperature field theory [5] [7].

Employing the diagrammatic CTP rules potential 'pinch singularities' might arise in strictly perturbative expressions. As an example we consider a scalar field theory. A typical contribution arising in a perturbative expansion takes e.g. the form

$$
D_{0}^{\text {ret }}\left(\vec{p}, p_{0}\right) \Sigma_{0}\left(\vec{p}, p_{0}\right) D_{0}^{\text {av }}\left(\vec{p}, p_{0}\right) .
$$

Here $\Sigma_{0}$ describes some physical (perturbative) quantity (e.g. a self energy insertion); $D_{0}^{\text {ret }}$ and $D_{0}^{\text {av }}$ denote the free retarded and advanced propagator, respectively. As $D_{0}^{\text {ret }}$ contains a pole at $p_{0}= \pm E_{p}-i \epsilon$ and $D_{0}^{\text {av }}$ a pole at $p_{0}= \pm E_{p}+i \epsilon$ the product of both in the above expression is ill-defined, if $\Sigma_{0}\left(\vec{p}, p_{0}=E_{p}=\sqrt{m^{2}+\vec{p}^{2}}\right)$ does not vanish onshell. Transforming such an expression back into a time representation, the contour has to pass between this pair of two infinitely close poles.

It was observed and proven by Landsman and van Weert that such ill-defined terms cancel each other in each order in perturbation theory, if the system stays at thermal equilibrium [6]. Their arguments, however, rely solely on the KMS boundary conditions of the free propagators and self energy insertions, so that they do not apply for systems out of equilibrium. This severe problem arising for systems out of equilibrium was first raised by Altherr and Seibert [8]. Indeed, it was speculated there that the CTP formalism might not be adequate for describing non-equilibrium systems at all. In a subsequent paper, Altherr [9] tried to 'cure' this problem by hand by introducing a finite width for the 'unperturbed' free CTP propagator $D_{0}$ so that the expressions are at least well-defined in a mathematical sense. Within his modified perturbative approach, he also showed that seemingly higher order diagrams do contribute to a lower order in the coupling constant, as some of the higher order diagrams involving pinch terms will receive factors of the form $1 / \Gamma^{n}, n \geq 1$ reducing substantially the overall power in the coupling constant. In his particular case Altherr investigated the dynamically generated effective mass (the 'tadpole' contribution) within standard $\phi^{4}$-theory. (For the hard modes the onshell damping $\Gamma$ is of the order of $o\left(g^{4} T\right)$.) Therefore he concluded that power counting arguments might in fact be much less trivial for systems out of equilibrium. We will come back to his observation below.

In a recent work [10] we have discussed in detail that modes or quasi-particles become thermally populated by a non-perturbative Langevin like interplay between noise and dissipative terms entering the non-equilibrium quantum transport equations. In the process of thermalization the full propagators necessarily must acquire some finite width (due to collisions or more generally due to damping). Plasmons behave as 'nonshell' modes [11]. Strictly speaking, the evolution of a non-equilibrium system towards equilibrium is always non-perturbative. We will come back to this interpretation in more detail below.

First, however, we will elaborate on the physical reason for the occurrence of pinch singularities in a strictly perturbative expansion, when an interacting system is prepared 
with some non-equilibrium occupation of the particles. As a motivation we were inspired by the idea that in principle the Schwinger-Keldysh formalism is also adequate to describe simple scattering processes where e.g. only two initial particles are prepared at some fixed momentum states in the past. Hence, the perturbative scheme of the Schwinger-Keldysh formalism should give the same results as elementary scattering theory.

To set the stage we start with some formulae and manipulations already presented in [8]. We follow the notation of [10]. For simplicity we consider in the following a weakly interacting scalar $\phi^{4}$-theory. The initial state in the far past (assuming a homogeneous and stationary system) is prepared by specifying the momentum occupation number $\tilde{n}(\vec{p})$ of the (initially non interacting) onshell particles. Note that this occupation number depends only on the three momentum $\vec{p}$. (If specified with a thermal equilibrium distribution at some given temperature, $\tilde{n}$ would be replaced by the onshell Bose distribution $n_{B}\left(E_{p}=\sqrt{m^{2}+\vec{p}^{2}}\right)$.) The occupation number $\tilde{n}(\vec{p})$ enters the (free) propagator

$$
D_{0}^{<}(p)=-2 \pi i \operatorname{sgn}\left(p_{0}\right) \delta\left(p^{2}-m^{2}\right)\left[\Theta\left(p_{0}\right) \tilde{n}(\vec{p})-\Theta\left(-p_{0}\right)(1+\tilde{n}(\vec{p}))\right]
$$

In addition, we note the form of the free retarded and advanced propagator:

$$
D_{0}^{\mathrm{ret} / \mathrm{av}}(p)=\frac{1}{p^{2}-m^{2} \pm i \epsilon \operatorname{sgn}\left(p_{0}\right)}
$$

To calculate perturbative corrections to the propagators we apply the Langreth-Wilkins rules 12] which are quite well-known within the context of the Schwinger-Keldysh formalism:

$$
\begin{aligned}
D^{\mathrm{ret}} & =D_{0}^{\mathrm{ret}}+D_{0}^{\mathrm{ret}} \Sigma_{0}^{\mathrm{ret}} D_{0}^{\mathrm{ret}}+\ldots=: D_{0}^{\mathrm{ret}}+\Delta D^{\mathrm{ret}} \\
D^{\mathrm{av}} & =D_{0}^{\mathrm{av}}+D_{0}^{\mathrm{av}} \Sigma_{0}^{\mathrm{av}} D^{\mathrm{av}}+\ldots=: D_{0}^{\mathrm{av}}+\Delta D^{\mathrm{av}}, \\
D^{<} & =D_{0}^{<}+D_{0}^{\mathrm{ret}} \Sigma_{0}^{\mathrm{ret}} D_{0}^{<}+D_{0}^{\mathrm{ret}} \Sigma_{0}^{<} D_{0}^{\mathrm{av}}+D_{0}^{<} \Sigma_{0}^{\mathrm{av}} D_{0}^{\mathrm{av}}+\ldots=: D_{0}^{<}+\Delta D^{<},
\end{aligned}
$$

where the dots denote multiple self energy insertions which we will not consider for the moment. Here the retarded and advanced self energies are given by the Fourier transforms of (cf. e.g. [10])

$$
\begin{aligned}
& \Sigma^{\mathrm{ret}}\left(x_{1}, x_{2}\right):=\Theta\left(t_{1}-t_{2}\right)\left[\Sigma^{>}\left(x_{1}, x_{2}\right)-\Sigma^{<}\left(x_{1}, x_{2}\right)\right], \\
& \Sigma^{\mathrm{av}}\left(x_{1}, x_{2}\right):=\Theta\left(t_{2}-t_{1}\right)\left[\Sigma^{<}\left(x_{1}, x_{2}\right)-\Sigma^{>}\left(x_{1}, x_{2}\right)\right] .
\end{aligned}
$$

The self energies $\Sigma^{>}$and $\Sigma^{<}$are related as $\Sigma^{>}\left(x_{1}, x_{2}\right)=\Sigma^{<}\left(x_{2}, x_{1}\right)$ in case of a scalar field theory. The self energy insertion $\Sigma_{0}$ in a strictly perturbative expansion is given by a convolution of the initial free propagators. If the initial momentum distribution entering the propagator (2) is given by the Bose equilibrium distribution, the important relation

$$
\Sigma^{>}(p)=e^{p_{0} / T} \Sigma^{<}(p),
$$

holds, which is nothing but the KMS boundary condition. It is worth mentioning that our conventions are chosen such that $i \Sigma^{<}(p)$ is always real and non negative. In a transport theory (see below) it can be interpreted as the production rate for modes with the respective energy. As a characteristic example we discuss in the following the 'sunset' graph arising in scalar $\phi^{4}$-theory. This diagram is illustrated in fig. 1. We choose this particular graph 
as an example since the self energies $\Sigma_{0}^{</>}\left(\vec{p}, p_{0}=E_{p}\right)$ do not vanish onshell for thermal distributions (see e.g. [13,14]). This also holds for any non-equilibrium distribution $\tilde{n}$ as long as the individual two-particle scattering contributions are kinematically allowed. Within finite temperature field theory the imaginary part of the self energy ('cut' diagram) taken onshell is connected to the scattering rate (as an illustration see fig. 2). On the other hand, there exist certain self energy insertions like the so-called hard thermal loop self energy 15 or other one-loop diagrams [16] which vanish on-shell due to simple kinematical constraints and thus do not cause any pinch problem.

By inspecting (4) more closely one finds that the perturbative corrections $\Delta D^{\text {ret/av }}$ to the free retarded/advanced propagator are free of any pinch singularities as the emerging poles are all located at the same side of the contour. We note in passing that this also holds for multiple self energy insertions in (口, 5] (see e.g. [10]). In contrast, all three contributions to $\Delta D^{<}$are ill-defined. Using the identity

$$
\pi \operatorname{sgn}\left(p_{0}\right) \delta\left(p^{2}-m^{2}\right)=\frac{i}{2}\left(D_{0}^{\mathrm{ret}}(p)-D_{0}^{\mathrm{av}}(p)\right)
$$

together with (2) we can further manipulate the three contributions of $\Delta D^{<}$by employing the Fourier transforms of the definitions (7) and (8). We find

$$
\Delta D^{<}(p)=\Delta D_{\text {reg }}^{<}(p)+\Delta D_{\text {pinch }}^{<}(p)
$$

with a regular part,

$$
\begin{aligned}
\Delta D_{\text {reg }}^{<}(p)=[ & \left.\Theta\left(p_{0}\right) \tilde{n}(\vec{p})-\Theta\left(-p_{0}\right)(1+\tilde{n}(\vec{p}))\right] \\
& \times\left(D_{0}^{\mathrm{ret}}(p) \Sigma_{0}^{\mathrm{ret}}(p) D_{0}^{\mathrm{ret}}(p)-D_{0}^{\mathrm{av}}(p) \Sigma_{0}^{\mathrm{av}}(p) D_{0}^{\mathrm{av}}(p)\right),
\end{aligned}
$$

and the part carrying the pinch singularities,

$$
\begin{aligned}
& \Delta D_{\text {pinch }}^{<}(p)=D_{0}^{\text {ret }}(p) {\left[\Theta\left(p_{0}\right)\left((1+\tilde{n}(\vec{p})) \Sigma_{0}^{<}(p)-\tilde{n}(\vec{p}) \Sigma_{0}^{>}(p)\right)\right.} \\
&\left.+\Theta\left(-p_{0}\right)\left((1+\tilde{n}(\vec{p})) \Sigma_{0}^{>}(p)-\tilde{n}(\vec{p}) \Sigma_{0}^{<}(p)\right)\right] D_{0}^{\mathrm{av}}(p) .
\end{aligned}
$$

The last expression is ill-defined, if the terms in the square brackets do not vanish onshell as already pointed out in [8]. The expression in the square brackets is familiar from standard kinetic theory (see e.g. [8, 10]): Apart from a trivial factor one can interpret

$$
\Gamma_{\mathrm{eff}}(\vec{p}):=\left.\frac{1}{2 E_{p}}\left[(1+\tilde{n}(\vec{p})) i \Sigma_{0}^{>}(p)-\tilde{n}(\vec{p}) i \Sigma_{0}^{<}(p)\right]\right|_{p_{0}=E_{p}}
$$

as the net effective rate for the change of the occupation number per time. For an equilibrium situation the occupation number is given by the Bose distribution and the self energy insertions fulfill the KMS condition (9). Hence, for the equilibrium case the whole bracket exactly vanishes and no pinch singularities emerge. In contrast, this is not the case for a general non-equilibrium configuration [8].

To shed first some light on the physical interpretation of this ill-defined expression one has to ask for observables which are affected by this singularity. Within standard scattering theory one would think about the probability for a particle of some initial momentum state 
to be scattered into another momentum state. Therefore we ask, how the occupation number $\tilde{n}$ has changed after a long time. The occupation number for the out-states can be readily extracted from $D^{<}$by means of the formula (for a derivation see [10])

$$
\begin{aligned}
& n(\vec{p}, t \rightarrow \infty)^{\text {(out) }}=\left\langle a_{\vec{p}}^{\dagger \text { (out) }} a_{\vec{p}}^{\text {(out) }}\right\rangle \\
& \quad=\left.\left(\frac{E_{p}}{2}+\frac{1}{2 E_{p}} \frac{\partial}{\partial t} \frac{\partial}{\partial t^{\prime}}+\frac{i}{2}\left(\frac{\partial}{\partial t}-\frac{\partial}{\partial t^{\prime}}\right)\right) \frac{1}{V} \int d^{3} x \int d^{3} y e^{i \vec{p} \vec{x}} e^{-i \vec{p} \vec{y}}\left(i D^{<}\left(\vec{y}, t ; \vec{x}, t^{\prime}\right)\right)\right|_{t^{\prime}=t} \\
& \stackrel{t \rightarrow \infty}{=} \int \frac{d p_{0}}{2 \pi}\left(\frac{E_{p}}{2}+\frac{p_{0}^{2}}{2 E_{p}}+p_{0}\right)\left(i D^{<}\left(\vec{p}, p_{0}\right)\right) .
\end{aligned}
$$

When inserting (11) one finds by contour integration that $\Delta D_{\text {reg }}^{<}$only yields a finite contribution. The same holds true for the $\Theta\left(-p_{0}\right)$-term in (13) since the 'particle projector' $\left(\frac{E_{p}}{2}+\frac{p_{0}^{2}}{2 E_{p}}+p_{0}\right)$ vanishes on the antiparticle mass shell. However, the $\Theta\left(p_{0}\right)$-term of the ill-defined expression $\Delta D_{\text {pinch }}^{<}$gives rise to the following infinite expression

$$
\Delta n(\vec{p})^{(\text {out })}=\Gamma_{\text {eff }}(\vec{p}) \cdot 2 \pi \delta(0)+\text { finite contributions. }
$$

From analogy to the standard derivation of Fermi's golden rule in elementary quantum scattering theory one is immediately tempted to interpret this $\delta(0)$ singularity as the elapsed scattering time $T \rightarrow \infty$. Indeed, this interpretation has very recently been conjectured by Niegawa in [17], where he was also elaborating on the issue of pinch singularities in nonequilibrium quantum field theory. His major point, however, has been to interpret the infinite shift $\Delta n(\vec{p})$ as a renormalization in the number density. We think, however, that this latter interpretation further obscures the problem instead of uncovering the physical processes which are at the bottom of the pinch problem.

To demonstrate that the pinch singularities indeed appear as a result of Fermi's golden rule in scattering theory we now assume that the interaction is switched on at a time $t=-T / 2$ and switched off at $t=T / 2$, i.e. we replace

$$
\Sigma_{0}^{</>}\left(x_{1}, x_{2}\right) \rightarrow \bar{\Sigma}_{0}^{</>}\left(x_{1}, x_{2}\right):=\Theta\left(\frac{T}{2}-t_{1}\right) \Theta\left(\frac{T}{2}-t_{2}\right) \Sigma_{0}^{</>}\left(x_{1}, x_{2}\right) \Theta\left(t_{1}+\frac{T}{2}\right) \Theta\left(t_{2}+\frac{T}{2}\right)
$$

and assume that the duration time $T$ is large but finite. This procedure regulates the pinch singularity to a finite value. As a first step we again extract the pinch term from (6), now working in the representation of three-momentum and time:

$$
\begin{aligned}
& \Delta D_{\text {pinch }}^{<}\left(\vec{p}, t, t^{\prime}\right) \\
& =\int \frac{d p_{0(1)}}{2 \pi} \frac{d p_{0(2)}}{2 \pi} \frac{d p_{0(3)}}{2 \pi} e^{-i p_{0(1)} t} e^{i p_{0(3)} t^{\prime}} D_{0}^{\mathrm{ret}}\left(\vec{p}, p_{0(1)}\right) D_{0}^{\mathrm{av}}\left(\vec{p}, p_{0(3)}\right) \\
& \quad \times\left[\left(\Theta\left(p_{0(1)}\right) \tilde{n}(\vec{p})-\Theta\left(-p_{0(1)}\right)(1+\tilde{n}(\vec{p}))\right) \Sigma_{0}^{\mathrm{av}}\left(\vec{p}, p_{0(2)}\right)+\Sigma_{0}^{<}\left(\vec{p}, p_{0(2)}\right)\right. \\
& \left.\quad-\Sigma_{0}^{\mathrm{ret}}\left(\vec{p}, p_{0(2)}\right)\left(\Theta\left(p_{0(3)}\right) \tilde{n}(\vec{p})-\Theta\left(-p_{0(3)}\right)(1+\tilde{n}(\vec{p}))\right)\right] \\
& \quad \times \int_{-T / 2}^{T / 2} d \bar{t} e^{i \bar{t}\left(p_{0(1)}-p_{0(2)}\right)} \int_{-T / 2}^{T / 2} d \bar{t}^{\prime} e^{i \bar{t}^{\prime}\left(p_{0(2)}-p_{0(3)}\right)} .
\end{aligned}
$$


As

$$
\int_{-T / 2}^{T / 2} d t e^{i t \Delta p}=\frac{2}{\Delta p} \sin \left(\frac{T}{2} \Delta p\right) \stackrel{T \rightarrow \infty}{\rightarrow} 2 \pi \delta(\Delta p)
$$

it becomes clear how the pinch singularity arises for $T \rightarrow \infty$. Furthermore, if $T$ is already sufficiently large, we are safely allowed to approximate $p_{0(1)} \approx p_{0(2)} \approx p_{0(3)}$ within the square bracket in $(\sqrt{19})$ :

$$
\begin{aligned}
{[\ldots] \approx[} & \Theta\left(p_{0(2)}\right)\left((1+\tilde{n}(\vec{p})) \Sigma_{0}^{<}\left(\vec{p}, p_{0(2)}\right)-\tilde{n}(\vec{p}) \Sigma_{0}^{>}\left(\vec{p}, p_{0(2)}\right)\right) \\
& \left.+\Theta\left(-p_{0(2)}\right)\left((1+\tilde{n}(\vec{p})) \Sigma_{0}^{>}\left(\vec{p}, p_{0(2)}\right)-\tilde{n}(\vec{p}) \Sigma_{0}^{<}\left(\vec{p}, p_{0(2)}\right)\right)\right] .
\end{aligned}
$$

We proceed by calculating $\Delta n(\vec{p})^{\text {(out) }}$ by means of (15). For this we first take $t, t^{\prime}>T / 2$, evaluate the $p_{0(1)^{-}}$and $p_{0(3)}$-integration by standard complex contour integration and then insert the emerging expression into (15). It results in

$$
\begin{aligned}
& \Delta n(\vec{p})_{\text {pinch }}^{\text {(out) }}=(-i)\left(\frac{E_{p}}{2}+\frac{1}{2 E_{p}} \frac{\partial}{\partial t} \frac{\partial}{\partial t^{\prime}}+\frac{i}{2}\left(\frac{\partial}{\partial t}-\frac{\partial}{\partial t^{\prime}}\right)\right) \\
& \otimes \int \frac{d p_{0(2)}}{2 \pi}\left[\Theta\left(p_{0(2)}\right)\left((1+\tilde{n}(\vec{p})) \Sigma_{0}^{<}\left(\vec{p}, p_{0(2)}\right)-\tilde{n}(\vec{p}) \Sigma_{0}^{>}\left(\vec{p}, p_{0(2)}\right)\right)\right. \\
& \left.+\Theta\left(-p_{0(2)}\right)\left((1+\tilde{n}(\vec{p})) \Sigma_{0}^{>}\left(\vec{p}, p_{0(2)}\right)-\tilde{n}(\vec{p}) \Sigma_{0}^{<}\left(\vec{p}, p_{0(2)}\right)\right)\right] \\
& \times \frac{1}{E_{p}}\left[\frac{\sin \left(\frac{T}{2}\left(p_{0(2)}+E_{p}\right)\right)}{p_{0(2)}+E_{p}} e^{i E_{p} t}-\frac{\sin \left(\frac{T}{2}\left(p_{0(2)}-E_{p}\right)\right)}{p_{0(2)}-E_{p}} e^{-i E_{p} t}\right] \\
& \times\left.\frac{1}{E_{p}}\left[\frac{\sin \left(\frac{T}{2}\left(p_{0(2)}-E_{p}\right)\right)}{p_{0(2)}-E_{p}} e^{i E_{p} t^{\prime}}-\frac{\sin \left(\frac{T}{2}\left(p_{0(2)}+E_{p}\right)\right)}{p_{0(2)}+E_{p}} e^{-i E_{p} t^{\prime}}\right]\right|_{t^{\prime}=t} \\
& \approx \Gamma_{\mathrm{eff}}(\vec{p}) \int \frac{d p_{0(2)}}{2 \pi} \frac{4}{\left(p_{0(2)}-E_{p}\right)^{2}} \sin ^{2}\left(\frac{T}{2}\left(p_{0(2)}-E_{p}\right)\right)=\Gamma_{\mathrm{eff}}(\vec{p}) \cdot T
\end{aligned}
$$

which is valid for large but finite $T$.

Thus we have demonstrated the bridge between the occurrence of pinch singularities within the context of the CTP formalism and Fermi's golden rule in elementary quantum scattering theory. The effective rate $\Gamma_{\text {eff }}$ is therefore analogous to the transition probability per unit time. Indeed one can easily understand in physical terms that one has to expect such a singularity in perturbation theory: Staying strictly within the first order contribution the particles remain populated with the initially prepared non-equilibrium occupation number (since this quantity enters the free propagator (2)) and scatter for an infinitely long time. Therefore, the resulting shift $\Delta n(\vec{p})^{\text {(out) }}$ should scale with $\Gamma_{\text {eff }}(\vec{p}) \cdot T$ with $\Gamma_{\text {eff }}(\vec{p})$ held fixed. We conclude that the occurrence of pinch singularities appearing in perturbative contributions within non-equilibrium quantum field theory is of no mystery, but actually it has to appear because of a very intuitive reason: the interaction time $T$ becomes infinite. However, looking at a Boltzmann equation which describes the time evolution of the particle distribution function in the semiclassical regime (see (30) below) one realizes that the occupation number does not stay constant during the dynamical evolution of the system, but 
will be changed on a timescale of roughly $1 / \Gamma$. The quasi-particles are not really asymptotic states.

Next, however, we will show how pinch singularities are formally cured by a resummation procedure. The onshell non-equilibrium effective rate $\Gamma_{\text {eff }}$ can be visualized as being the net result of collisions between the onshell particles. From standard thermal field theory one would thus expect that the propagators will become dressed and supplemented by a finite (collisional or more generally damping) width. This represents already a non-perturbative effect which only can be achieved by a resummation of Dyson-Schwinger type. As a first attempt (proposed by Baier et al. [18]), one might resum the full series of (因囵) using the self energy $\Sigma_{0}$ (recall that the latter is calculated from free propagators):

$$
D=D_{0}+D_{0} \Sigma_{0} D_{0}+D_{0} \Sigma_{0} D_{0} \Sigma_{0} D_{0}+\ldots=D_{0}+D_{0} \Sigma_{0} D .
$$

With the definitions $\Gamma_{0}\left(\vec{p}, p_{0}\right):=\frac{i}{2 p_{0}}\left[\Sigma_{0}^{>}\left(\vec{p}, p_{0}\right)-\Sigma_{0}^{<}\left(\vec{p}, p_{0}\right)\right]$ and $\operatorname{Re} \Sigma_{0}:=\operatorname{Re} \Sigma_{0}^{\mathrm{ret}}=\operatorname{Re} \Sigma_{0}^{\mathrm{av}}$ we end up with (cf. e.g. [10])

$$
\begin{aligned}
D^{\mathrm{ret}} & =D_{0}^{\mathrm{ret}}+D_{0}^{\mathrm{ret}} \Sigma_{0}^{\mathrm{ret}} D^{\mathrm{ret}}=\frac{1}{p^{2}-m^{2}-\operatorname{Re} \Sigma_{0}+i p_{0} \Gamma_{0}}, \\
D^{\mathrm{av}} & =D_{0}^{\mathrm{av}}+D_{0}^{\mathrm{av}} \Sigma_{0}^{\mathrm{av}} D^{\mathrm{av}}=\frac{1}{p^{2}-m^{2}-\operatorname{Re} \Sigma_{0}-i p_{0} \Gamma_{0}}, \\
D^{<} & =D^{\mathrm{ret}} \Sigma_{0}^{<} D^{\mathrm{av}}=(-2 i) \frac{p_{0} \Gamma_{0}}{\left(p^{2}-m^{2}-\operatorname{Re} \Sigma_{0}\right)^{2}+p_{0}^{2} \Gamma_{0}^{2}} \frac{\Sigma_{0}^{<}}{\Sigma_{0}^{>}-\Sigma_{0}^{<}} .
\end{aligned}
$$

Hence the resummation of the series (6) of ill-defined terms results in a well-defined expression. The quantity

$$
n_{\Sigma}\left(\vec{p}, p_{0}\right):=\frac{\Sigma_{0}^{<}}{\Sigma_{0}^{>}-\Sigma_{0}^{<}}
$$

appearing in (26) has to be interpreted as the 'occupation number' demanded by the self energy parts [10]. If the equilibrium KMS conditions (9) apply for the self energy part, then $n_{\Sigma}\left(\vec{p}, p_{0}\right) \stackrel{\mathrm{KMS}}{\longrightarrow} n_{B}\left(p_{0}\right)$ becomes just the Bose distribution function. For a general non-equilibrium situation, however, this factor deviates from the Bose distribution. If the damping width is sufficiently small, i.e. $\bar{\Gamma}, \bar{\Sigma}>, \bar{\Sigma}^{<}$are proportional to some power in the (small) coupling constant $g$ (e.g. $\sim g^{4}$ in case of the sunset graph depicted in fig. 便) the expression (26) results in

$$
D^{\mathrm{ret}} \Sigma_{0}^{<} D^{\mathrm{av}} \stackrel{g \rightarrow 0}{\longrightarrow}-2 \pi i \operatorname{sgn}(\omega) \delta\left(p^{2}-m^{2}\right) \lim _{g \rightarrow 0} n_{\Sigma}\left(\vec{p}, p_{0}\right) .
$$

When evaluating the occupation number for the out-states by means of (16) one accordingly will get

$$
n(\vec{p})^{\text {(out) }} \approx n_{\Sigma}\left(\vec{p}, E_{p}\right)
$$

which is free of any pathological behavior. The astonishing thing to note at this point is that in fact the (initial) non-equilibrium distribution $\tilde{n}$ has been substituted by $n_{\Sigma}$ and, therefore, does not show up explicitly. So the question is, how $\tilde{n}$ enters? 
Calculating $\Sigma_{0}$ on a purely perturbative level the initial occupation number $\tilde{n}$ enters via the free propagator (2). This however cannot be the whole truth in a dynamically evolving system. It is important to make sure that such a system is prepared at some finite initial time $t_{0}$. (If $t_{0}$ would be taken as $t_{0} \rightarrow-\infty$ the system would already have reached equilibrium long time ago.) Bedaque [19] already has noted that pinch singularities are in fact an artifact of the boundaries chosen at $t_{0} \rightarrow-\infty$. Time reversal symmetry is explicitly broken, so that the propagators in principle have to depend on both time arguments explicitly before the system has reached a final equilibrium configuration. Therefore the use of Fourier techniques (which in fact has led to the pinch singularities in (13)) is highly dubious. The initial out of equilibrium distribution $\tilde{n}\left(t_{0}\right)$ cannot stay constant during the evolution of the system as it has to evolve towards the Bose distribution. Hence there must exist contributions which attribute to the temporal change of the distribution function. As long as the system is not in equilibrium (on a time scale of roughly $1 / \Gamma_{0}\left(\vec{p}, E_{p}\right)$ ), the propagator thus cannot be stationary. In addition, the self energy parts $\Sigma^{<}$and $\Sigma^{>}$do also evolve with time. Hence they should depend on the evolving distribution function and not persistently on the initial one, $\tilde{n}$, which enters $\Sigma_{0}$ in (23). Thus the resummation of (23) does not cover all relevant contributions. Speaking more technically, the self energy operators must also be evaluated consistently by the fully dressed and temporally evolving one-particle propagators.

The solution to these demands is, of course, the description of the system by means of appropriate (quantum) transport equations [20,4, 10]. Graphically this is illustrated in fig. 3 . In addition to the sunset diagram we have also included the mean field or Hartree diagram there which in a perturbative scheme is the one which arises first. (It would, however, not result in a pinch singularity so that we had discarded it in our previous discussion.) The difference to the resummation of $(23)$ is the fact that the propagators entering into the self-energy operators are now also the fully dressed ones. Such a skeleton expansion of the self energies with including the dressed propagators in the resummation is also familiar in standard quantum many-body theory for strongly interacting systems [21].

Unfortunately, the full quantum transport equations are generally hard to solve and thus are not so much of practical use. Yet one need not be that pessimistic. If the coupling is weak, i.e. the damping width is sufficiently small compared to the quasi-particle energy (which one typically assumes for many applications) one can take the Markov approximation to obtain standard kinetic equations (for a derivation see e.g. [20,4, 10]). For the situation illustrated in fig. 3 one gets the standard form [10]

$$
\begin{aligned}
& \left(E_{p} \partial_{t}-\vec{p} \partial_{\vec{x}}-\partial_{\vec{x}} m(\vec{x}, t) \partial_{\vec{p}}\right) f(\vec{x}, t ; \vec{p}) \\
& =\frac{1}{2}\left[i \Sigma^{<}\left(\vec{x}, t ; \vec{p}, E_{p}\right)(f(\vec{x}, \vec{p}, t)+1)-i \Sigma^{>}\left(\vec{x}, t ; \vec{p}, E_{p}\right) f(\vec{x}, \vec{p}, t)\right]
\end{aligned}
$$

Here $f$ denotes the semi-classical non-equilibrium phase-space distribution of quasi-particles. $m(\vec{x}, t)$ denotes the sum of the bare and the dynamical (space time dependent) mass generated by the Hartree term. Within the spirit of kinetic theory one easily realizes that the result obtained in (22) simply states that the change in the occupation number per time $T$ is nothing but the collision rate. Within this Markovian approximation the fully dressed propagators are given by 10

$$
D^{\mathrm{ret}}(\vec{x}, t ; p) \approx \frac{1}{p^{2}-m^{2}(\vec{x}, t)-\operatorname{Re} \Sigma(\vec{x}, t ; p)+i p_{0} \Gamma(\vec{x}, t ; p)},
$$




$$
\begin{aligned}
D^{\mathrm{av}}(\vec{x}, t ; p) & \approx \frac{1}{p^{2}-m^{2}(\vec{x}, t)-\operatorname{Re} \Sigma(\vec{x}, t ; p)-i p_{0} \Gamma(\vec{x}, t ; p)} \\
D^{<}(\vec{x}, t ; p) & \approx(-2 i) \frac{p_{0} \Gamma(\vec{x}, t ; p)}{\left(p^{2}-m^{2}(\vec{x}, t)-\operatorname{Re} \Sigma(\vec{x}, t ; p)\right)^{2}+p_{0}^{2} \Gamma^{2}(\vec{x}, t ; p)} f(\vec{x}, t ; \vec{p}) .
\end{aligned}
$$

In particular we emphasize that in (33) the instantaneous non-equilibrium phase space distribution function $f(t)$ enters and not the initial one, $\tilde{n}$. The dynamically generated mass as well as the collisional self energy contribution $\Sigma$ can thus be evaluated with these propagators. (In kinetic theory one usually takes the propagators in their quasi-free limit $(\operatorname{Re} \Sigma$, $\Gamma \rightarrow 0$ ), albeit instantaneous.) Higher order terms leading to the pinch singularities are explicitly resummed and lead now to finite and very transparent results.

One can now easily understand the observations made by Altherr [9]. He has found, starting from some non-equilibrium distribution $\tilde{n}$, that higher order diagrams contribute to the same order in the coupling constant as the lowest order one. Indeed, in his investigation, the particular higher order diagrams where nothing but the perturbative contributions of the series in (6) for the dressed or resummed one-particle propagator $D^{<}$. The only difference is that he has employed a 'free' propagator modified by some finite width in order that each of the terms in the series (6) becomes well defined. The reason for the higher order diagrams to contribute to the same order is that the initial out-of-equilibrium distribution $\tilde{n}$ cannot stay constant during the evolution of the system as it has to evolve towards the Bose distribution. If $\tilde{n}-n_{B}$ is of order $o(1)$, it is obvious that there must exist contributions which perturbatively attribute to the temporal change of the distribution function and contribute to the same order $o(1)$. In fact, in our prescription (33), $\tilde{n}$ has simply be substituted by the actual phase space distribution $f$. Then calculating e.g. the tadpole diagram, as discussed in the particular case of [9], one has to stay within lowest order in the skeleton expansion, but with the fully dressed propagator.

In summary, we have shown in simple physical terms why so called pinch singularities do (and have to) appear in the perturbative evaluation of higher order diagrams within the CTP description of non-equilibrium quantum field theory. They are simply connected to the standard divergence in elementary scattering theory. The occurrence of pinch singularities signals the occurrence of (onshell) damping or dissipation. This necessitates in the description of the evolution of the system by means of non-perturbative transport equations. In the weak coupling regime this corresponds to standard kinetic theory. In this case we have given a prescription of how the dressed propagators can be approximated in a very transparent form. Technically, pinch singularities appear due to a misuse of Fourier techniques [19]. From a physical point of view, scattering processes which change the occupation number give rise to pinch singularities, if these processes go on for infinitely long time. However, exactly these processes drive the system towards thermal equilibrium within a finite time characterized by the inverse damping rate. In equilibrium the occupation number stays constant and no pinch singularities can appear.

\section{ACKNOWLEDGMENTS}

We gratefully acknowledge discussions with M. Thoma. 


\section{REFERENCES}

[1] J. Schwinger, J. Math. Phys. 2, 407 (1961).

[2] P.M. Bakshi and K.T. Mahanthappa, J. Math. Phys. 4, 1, 12 (1963).

[3] L.V. Keldysh, Zh. Eks. Teor. Fiz. 47, 1515 (1964); Sov. Phys. JETP 20, 1018 (1965).

[4] K. Chou, Z. Su, B. Hao and L. Yu, Phys. Rep. 118, 1 (1985).

[5] R. Mills, 'Propagators for Many Particle Systems', Gordon and Breach, New York (1969).

[6] N.P. Landsman and Ch.G. van Weert, Phys. Rep. 145, 141 (1987).

[7] M. Le Bellac, 'Thermal Field Theory', Cambridge Univ. Press, Cambridge (1996).

[8] T. Altherr and D. Seibert, Phys. Lett. B333, 149 (1994).

[9] T. Altherr, Phys. Lett. B341, 325 (1995).

[10] C. Greiner and S. Leupold, 'Stochastic interpretation of Kadanoff-Baym equations and their relation to Langevin processes', hep-ph/9802312, to appear in Ann. Phys.

[11] N.P. Landsman, Ann. Phys. 186, 141 (1988).

[12] D. Langreth and J.W. Wilkins, Phys. Rev. B6, 3189 (1972).

[13] E. Wang and U. Heinz, Phys. Rev. D53, 899 (1996).

[14] C. Greiner and B. Müller, Phys. Rev. D55, 1026 (1997).

[15] M.E. Carrington, H. Defu and M.H. Thoma, 'Equilibrium and Non-Equilibrium Hard Thermal Loop Resummation in the Real Time Formalism', hep-ph/9708363.

[16] I. Dadic, 'Two mechanisms for elimination of pinch singularities in out of equilibrium thermal field theories', hep-ph/9801399.

[17] A. Niegawa, Phys. Lett. B416, 137 (1998).

[18] R. Baier, M. Dirks, K. Redlich, Phys. Rev. D55, 4344 (1997); R. Baier, M. Dirks, K. Redlich and D. Schiff, Phys. Rev. D56, 2548 (1997).

[19] P.F. Bedaque, Phys. Lett. B344, 23 (1995).

[20] L.P. Kadanoff and G. Baym, 'Quantum Statistical Mechanics', Benjamin, New York (1962).

[21] A.L. Fetter and J.D. Walecka, 'Quantum Theory of Many Particle Systems', McGrawHill Inc. (1971). 


\section{FIGURES}

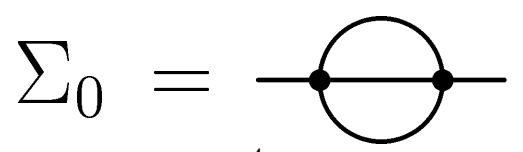

FIG. 1. Lowest order self energy term in $\phi^{4}$-theory which contributes to the pinch problem (sunset diagram).
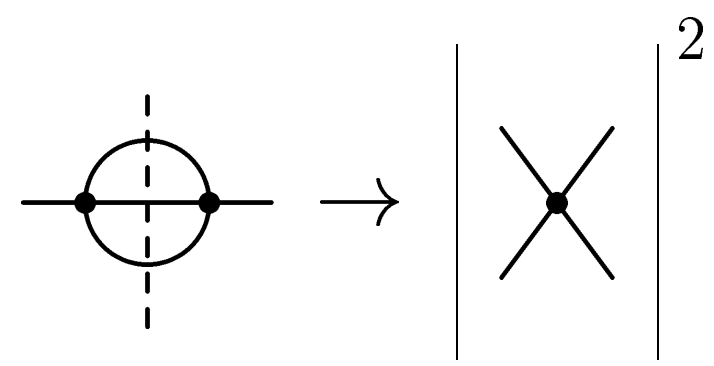

FIG. 2. Imaginary part of the sunset diagram which can be identified with a scattering amplitude.

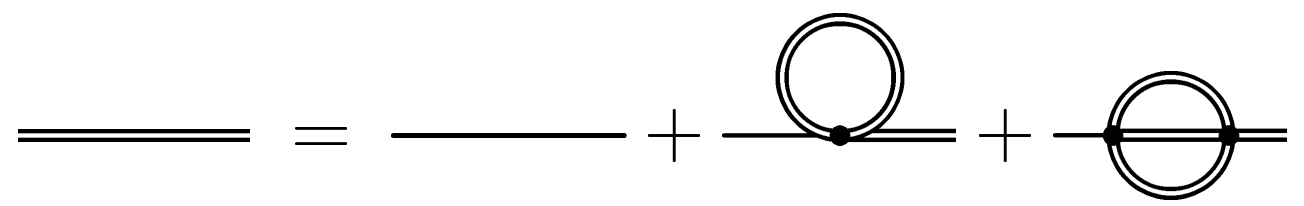

FIG. 3. Dyson-Schwinger equation with fully dressed propagators (skeleton expansion). 